\title{
Response of Two Maize Hybrids to Urea Fertilization under Application of Hydroquinone Urease Inhibitor
}

\author{
A.A. Mansour \\ Plant Production Department (Agronomy), Institute of Efficient \\ Productivity, Zagazig University, Sharkia, Egypt.
}

\begin{abstract}
TWO FIELD experiments were conducted during 2006 and 2007 summer seasons at Gaziret-Hanout,Kafr Sakr District, Sharkia Governorate, Egypt to study the response of two maize hybrids (SC 122 and TWC 310) to three levels of urea fertilization, i.e. 75,100 and $125 \mathrm{kgN} / \mathrm{fad}(1 \mathrm{ha}=2.381 \mathrm{fad})$ under application of three hydroquinone (HQ) urease inhibitor levels $(0,1.5$ and $3.0 \mathrm{~kg} \mathrm{HQ} / 100 \mathrm{~kg} \mathrm{~N})$. The adopted experimental design was split-split plot with three replicates.

The results revealed that TWC 310 maize hybrid was superior in most of studied characters compared to SC122 which superior in ear length and protein percentage. However, both tested hybrids gave almost the same protein yield/fad. Increasing $\mathrm{N}$ levels from 75 to $100 \mathrm{~kg} \mathrm{~N} / \mathrm{fad}$ resulted in a significant increase in plant height, 100-grain weight, protein percentage and protein yield/fad, while the increase in urea-N levels up to $125 \mathrm{kgN} / \mathrm{fad}$ increased significantly all other studied characters, except oil percentage which significantly decreased by any increase in $\mathrm{N}$ levels up to $125 \mathrm{kgN} /$ fad. Treating urea fertilizer with HQ urease inhibitor at the rate of $1.5 \mathrm{kgHQ} / 100 \mathrm{~kg} \mathrm{~N}$ increased significantly both plant height and 100-grain weight, while increasing $\mathrm{HQ}$ levels up to $3.0 \mathrm{kgHQ} / 100 \mathrm{~kg} \mathrm{~N}$ resulted in a significant increase in the other most studied characters compared with untreated urea. Any further HQ increment caused further reduction in oil percentage.
\end{abstract}

Regarding the interaction effects, TWC 310 gave the higher number of grains/ ear when urea was treated with $3.0 \mathrm{kgHQ} / 100 \mathrm{kgN}$ with a response rate of 25.66 grain yield/ fad was responded to urea-N up to $100 \mathrm{~kg} \mathrm{~N} / \mathrm{fad}$ when 1.5 or $3.0 \mathrm{~kg} \mathrm{HQ} / 100 \mathrm{~kg} \mathrm{~N}$ was added. The highest oil yield / fad was attained when $125 \mathrm{~kg} \mathrm{~N} / \mathrm{fad}$ was applied and when urea treated with $3.0 \mathrm{~kg} \mathrm{HQ} / 100 \mathrm{~kg} \mathrm{~N}$.

The results revealed positive and significant correlation coefficients between grain yield/ fad, and most of studied characters, while negative and significant correlation was found for grain yield related to oil percentage. The path analysis revealed that the direct effect of number of ears/plant was $17.06 \%$ being higher than that of 100-grain weight and number of grains/ear which was 15.18 and $5.11 \%$ of maize grain yield variation, respectively. Regression analysis revealed that number of ears/ plant and number of grains/ ear are similar to that of grain yield and each is of quadratic relationship with 
urea-N fertilization, while the response of 100-grain weight to urea-N fertilizer had a linear relationship.

Keywords: Maize, Urease inhibitor, Hydroquinone, N-fertilization, Yield analysis .

In Egypt, the local maize production is still not sufficient to meet the increase in consumption. The increase of maize grain yield could be achieved by using high yielding varieties suited to different environmental conditions and adopting optimum agronomic practices. Recently, Research Institutes provided farmers with commercial high yielding maize varieties which respond to high nitrogen fertilization doses (Aly et al.,1996).

Maize varieties differ in their yielding abilities depending on their genetic structures and their interaction with the environmental conditions. Many investigators found differences among the tested maize varieties, of them: Khamis et al. (2005), Oraby et al. (2005) and Abd El-Maksoud \& Sarhan (2008).

It is well known that nitrogen is a key element in maize nutrition. Therefore, an adequate supply of nitrogen is essential to maximize maize yield. Khamis et al. (2005) reported that ear length, number of ears/plant, number of grains/row, ear grain weight, 100-grain weight and grain yield/fad increased significantly with the increase in urea - $\mathrm{N}$ from 30 to $90 \mathrm{~kg} \mathrm{~N} / \mathrm{fad}$ with insignificant difference between 90 and $120 \mathrm{~kg} \mathrm{~N} / \mathrm{fad}$, while number of rows /ear did not respond significantly to the increase in urea - $\mathrm{N}$ rates. Oraby et al. (2005) found that number of grains per both row and ear, 100-grain weight as well as grain yield/fad were continuously and significantly increased by each increment of N-rate up to $150 \mathrm{~kg} / \mathrm{fad}$, while number of rows/ear was gradually increased up to the highest $\mathrm{N}$ rates in old lands and up to the medium $\mathrm{N}$ rate in new reclaimed lands. Also, Hans (2006), Abd El-Maksoud \& Sarhan (2008) and Thiraporn et al. (2008) came to the same conclusions in this respect.

Urea is one of the most commonly $\mathrm{N}$ - fertilizer used for maize production, but here in Egypt, most of farmers conventionally apply urea to soil surface. This practice can result in extreme loss of urea - $\mathrm{N}$ through $\mathrm{NH}_{3}$ voltalization (Khamis et al., 2005). One approach to decrease urea $\mathrm{N}$-loss via $\mathrm{NH}_{3}$ voltalization is the use of urease inhibitors that retard rapid urea hydrolysis and therefore reduce $\mathrm{NH}_{3}$ voltalization potential and this can result in increasing the response to nitrogen and hence, maize grain yield to increase.

Concerning urease inhibitors and its effects on decreasing urea - $\mathrm{N}$ loss via $\mathrm{NH}_{3}$ voltalization, Zhang et al. (1997) reported that adding dicyandiamide as an ammonia - stabilizing agent to ammonium bicarbonate as a nitrogen fertilizer caused a reduction of direct $\mathrm{NH}_{3}$ voltalizaton by $53 \%$, a fertilizer availability period prolonged from $35-45$ to $90-110$ days and an increase in the rate of nitrogen fertilizer being utilized by $5.9-10.2 \%$ and saving of the amount of fertilizer to be applied by $20-30 \%$ for the same level of maize grain yield, or an

Egypt. J. Agron. 34, No. 1 (2012) 
increase of maize grain yield by over $10 \%$ for the same level of nitrogen fertilizer. Hernan et al. (1999), found that ammonia losses from urea without adding the urease inhibitor $\mathrm{N}$-(n-butyl) thiophosphoric triamide ranged between 2.6 and $13.3 \%$ of applied N, being greater with higher N-rates. Further more, Khamis et al. (2005) detected a significant increase in ear length, ear number/ plant, row number/ ear, grain number / row, ear grain weight, 100-grain weight, grain yield / fad, $\mathrm{N}$-concentration in grains and $\mathrm{N}$-use efficiency of maize due to adding 1,4 - phenylenediamine (PDA) and hydroquinone (HQ) to urea fertilizer as urease inhibitors. However, no compound has been found to meet all the requirements for an ideal inhibitor of urease (Vetsch \& Randall, 2000).

This study aimed to investigate the response of two maize hybrids to urea fertilizer levels under urease inhibitor application.

\section{Materials and Methods}

Two field experiments were carried out at Gaziert -Hanout, Kafr Sakr District, Sharkia Governorate, Egypt during 2006 and 2007 summer seasons. The aim of this investigation was to study the response of two maize hybrids (SC 122 and TWC 310) to three urea fertilizer levels $(75,100$ and $125 \mathrm{~kg} \mathrm{~N} / \mathrm{fad})$ under three levels of hydroquinone (HQ) as urease inhibitor (0, 1.5 and $3.0 \mathrm{~kg} \mathrm{HQ} / 100 \mathrm{~kg} \mathrm{~N})$.

A split-split plot design with three replicates was used, where maize hybrids occupied the main plots. The three urea levels were allotted to the sub plots, whereas the HQ levels were randomly distributed in the sub-sub plots. The soil of the experimental field was loamy sand in texture having a $\mathrm{pH} 8.0$ and $8.1 ; 1.08$ and $1.10 \%$ organic matter content and containing 40.15 and 39.25 ppm available $\mathrm{N}, 9.18$ and $8.95 \mathrm{ppm}$ available $\mathrm{P}$ and 195 and $223 \mathrm{ppm}$ available $\mathrm{K}$ for the upper $30 \mathrm{~cm}$ of the soil surface in the two growing seasons, respectively. The plot area was $16.8 \mathrm{~m}^{2}$ included 6 ridges of $4 \mathrm{~m}$ long and $70 \mathrm{~cm}$ apart. The preceding crop in the two seasons was lupin.

Maize (Zea mays L.) seeds were sown on $3^{\text {rd }}$ June in both seasons with hill spacing of $25 \mathrm{~cm}$. Maize plants were thinned to one plant / hill 20 days after sowing and before the $2^{\text {nd }}$ irrigation. The urea fertilizer $(46.5 \% \mathrm{~N})$ was used in three equal doses, after 20, 40 and 60 days from sowing. Hydroquinone (HQ) urease inhibitor was adhered to urea prills by first dissolving the powdered HQ in methanol then spraying it on the urea prills just before urea application. Calcium superphosphate $\left(15.5 \% \mathrm{P}_{2} \mathrm{O}_{5}\right)$ at the rate of $100 \mathrm{~kg} / \mathrm{fad}$ and potassium sulphate $\left(48 \% \mathrm{~K}_{2} \mathrm{O}\right)$ at the rate of $50 \mathrm{~kg} / \mathrm{fad}$ were added as a basical fertilization just before sowing. The prevailing agronomic practices in the region were kept.

At harvest, ten guarded plants were taken from the $2^{\text {nd }}$ and $5^{\text {th }}$ ridges in each plot, then plant height $(\mathrm{cm})$, ear length $(\mathrm{cm})$, number of ears / plant, number of rows / ear, number of grains / row, number of grains / ear and 100-grain weight were measured. Grain yield (ardab/fad), which adjusted to $15.5 \%$ moisture content was determined from the central two ridges $\left(5.6 \mathrm{~m}^{2}\right)$ in each plot. To 
determine crude protein and oil contents, samples of dried grains were ground to fine powder and $\mathrm{N}$ content was determined using the modified micro-Kijeldahel apparatus as described by A.O.A.C. (1988). The obtained $\mathrm{N}$ values were multiplied by 6.25 to calculate crude protein percentage. Oil content was extract by diethylether in a soxhlet apparatus according to Comstock \& Culberston (1958). Protein and oil yields/ fad were calculated from multiplying grain yield / fad in $\mathrm{kg}$ by protein and oil percentages, respectively.

The proper statistical analysis of split - split plot design was used. Combined analysis was performed for the characters recorded in both seasons. Differences among treatment means were judjed using Duncan's multiple| range test (Duncan, 1955). Means followed by different letters were statistically significant. In interaction Tables, small and capital letters were used to compare means in columns and rows, respectively. The combined data of yield components and yield were subjected to simple correlation, path coefficient and regression analysis calculated according to Svab (1973).

\section{Results and Discussion}

\section{Yield and yield attributes}

Data presented in Tables 1, 2, 3 and 4 show the effect of both urea-N and urease inhibitor hydroquinone (HQ) levels on yield and its attributes in both growing seasons as well as the combined analysis of the two tested maize hybrids.

\section{Maize hybrid differences}

Combined analysis revealed that TWC 310 surpassed the other maize hybrid SC 122 in plant height, number of ears / plant, number of rows/ ear, number of grains/row, number of grains/ear, 100-grain weight, oil percentage and both grain and oil yields/ fad. The SC 122 produced the longer ears and the higher protein percentage compared to TWC 310 . However, both studied hybrids were insignificantly differed in protein yield/ fad. These differences between the two maize hybrids depending on the genetic make up and its interaction with the environmental conditions. Similar differences among maize hybrids were observed by Ahmed \& El-Sheikh (2002), Khamis et al. (2005), Oraby et al. (2005) and Abd El-Maksoud \& Sarhan (2008).

\section{Urea - N fertilization effects}

According to the combined data, ear length, number of ears /plant, number of rows/ear, number of grains / row, number of grains / ear and grain and oil yields/fad significantly increased by any increment of $\mathrm{N}$ fertilizer up to $125 \mathrm{~kg}$ $\mathrm{N} /$ fad. Whereas, plant height, 100-grain weight, protein percentage and protein yield/ fad significantly increased up to $100 \mathrm{~kg} \mathrm{~N} / \mathrm{fad}$. The mean increases of each increment up to $125 \mathrm{~kg} \mathrm{~N} / \mathrm{fad}$ were $0.885 \mathrm{ardab}, 8.925 \mathrm{~kg}$ and $13.195 \mathrm{~kg} /$ fad for grain, protein and oil yields, respectively. However, oil percentage significantly decreased by increasing $\mathrm{N}$-levels up to $125 \mathrm{~kg} \mathrm{~N} / \mathrm{fad}$. These findings are in agreement with those obtained by Ahmed \& El-Sheikh (2002), Mohamed (2004), Khamis et al. (2005), Oraby et al. (2005) and Abd El-Maksoud \& Sarhan (2008).

Egypt. J. Agron. 34, No. 1 (2012) 
RESPONSE OF TWO MAIZE HYBRIDS ...

\begin{tabular}{|c|c|c|c|c|c|}
\hline 혈 & 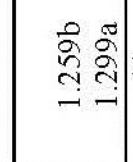 & 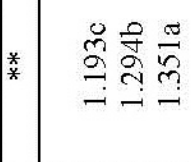 & $\stackrel{*}{*}$ & 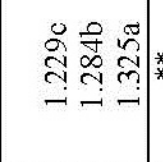 & $\begin{array}{lll}2 & 2 & 2 \\
z & z & z \\
z\end{array}$ \\
\hline 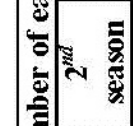 & 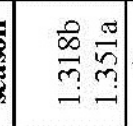 & 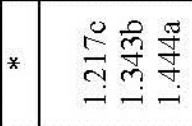 & $\frac{*}{*}$ & 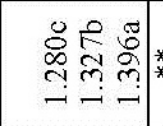 & 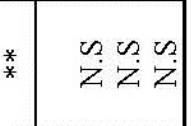 \\
\hline 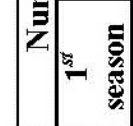 & $\mid \begin{array}{ll}0 \\
0 \\
0\end{array}$ & 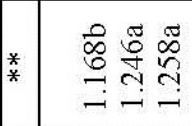 & $\underset{*}{*}$ & 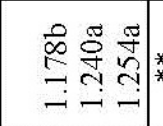 & 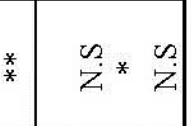 \\
\hline 豆 & 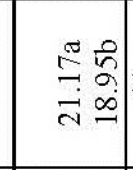 & 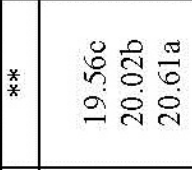 & $\frac{*}{*}$ & 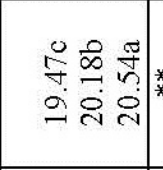 & 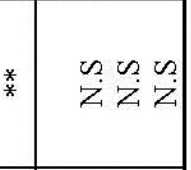 \\
\hline 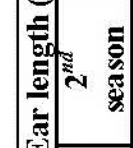 & 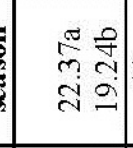 & 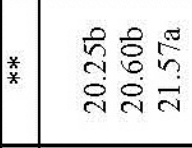 & $\stackrel{*}{*}$ & 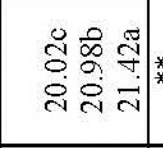 & 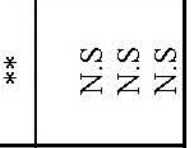 \\
\hline$=-\frac{\bar{t}}{2}$ & 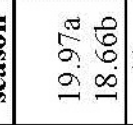 & 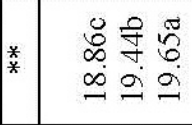 & $\frac{*}{*}$ & 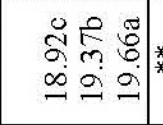 & 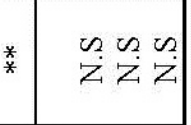 \\
\hline 药 & 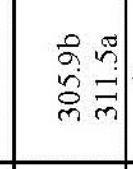 & 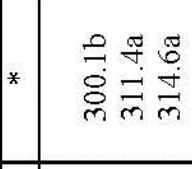 & $\stackrel{*}{*}$ & 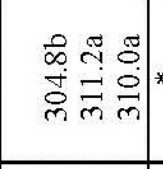 & 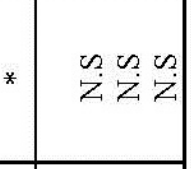 \\
\hline 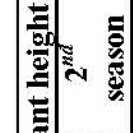 & 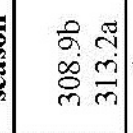 & 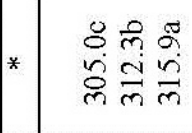 & $\frac{*}{*}$ & 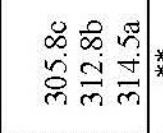 & $\begin{array}{lll}* & 2 & 0 \Omega \\
* & z & z\end{array}$ \\
\hline$=\frac{5}{20}$ & $\begin{array}{l}0 . \\
\text { लें } \\
\text { 。ें }\end{array}$ & 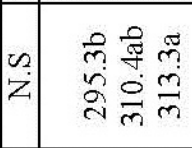 & ${ }_{*}^{*}$ & 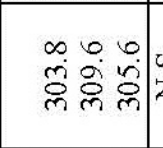 & 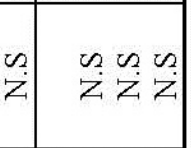 \\
\hline 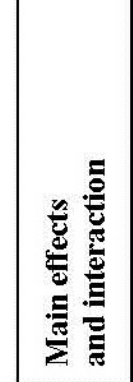 & 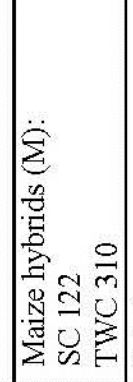 & 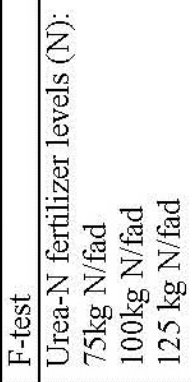 & $\overrightarrow{4}$ & 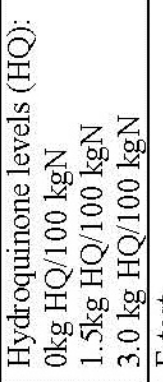 & 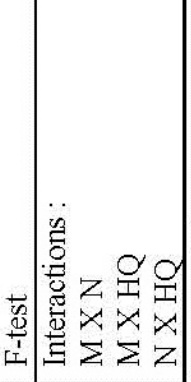 \\
\hline
\end{tabular}

Egypt. J. Agron. 34, No. 1 (2012) 


\begin{tabular}{|c|c|c|c|c|c|}
\hline 离 & 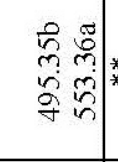 & 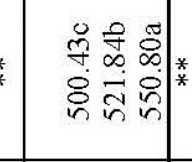 & 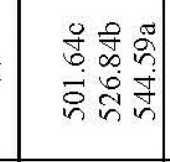 & $*$ & 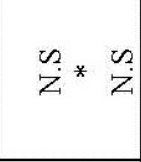 \\
\hline 就 & 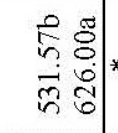 & 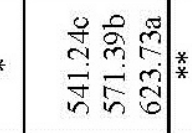 & 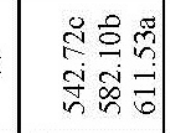 & $\frac{*}{*}$ & 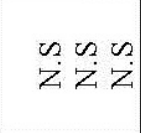 \\
\hline 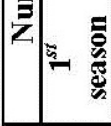 & 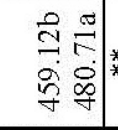 & 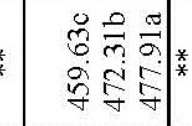 & 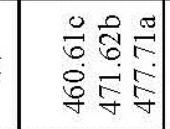 & $\frac{*}{*}$ & * $\begin{array}{l}* \\
* \\
z\end{array}$ \\
\hline 袅 & 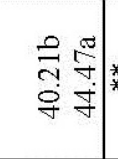 & 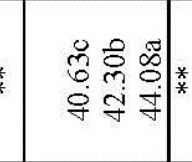 & 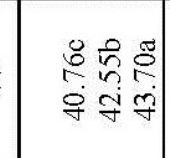 & $\stackrel{*}{*}$ & 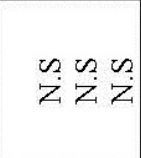 \\
\hline 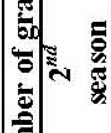 & 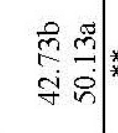 & 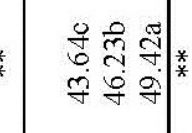 & 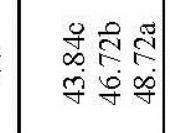 & $\frac{*}{*}$ & 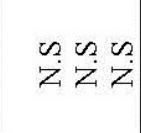 \\
\hline 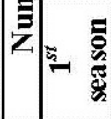 & 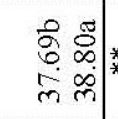 & 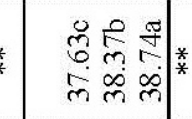 & $\begin{array}{lll} & 0 & 0 \\
0 & 0 & 0 \\
0 & m & 0 \\
c & 0 & 0 \\
m & m & 0\end{array}$ & * & 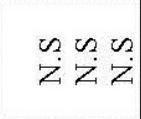 \\
\hline है & 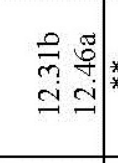 & 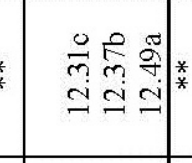 & 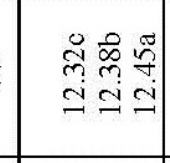 & $*$ & 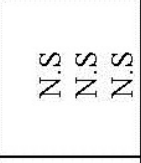 \\
\hline 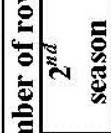 & 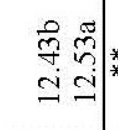 & 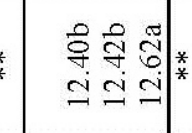 & 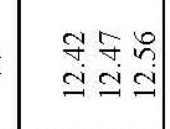 & $\mid$ & 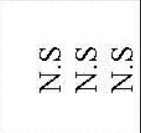 \\
\hline 妾 & 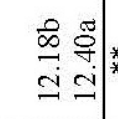 & 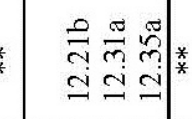 & 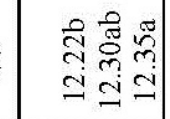 & $*$ & $* * \frac{\Omega}{z}$ \\
\hline 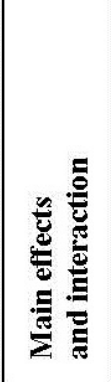 & 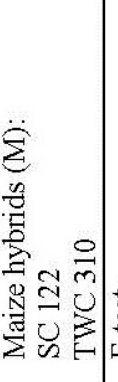 & 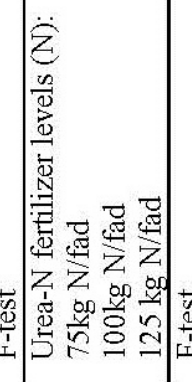 & 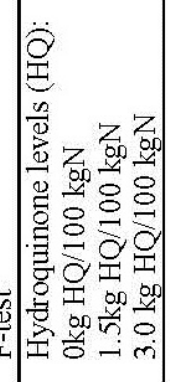 & 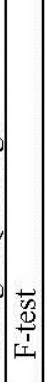 & 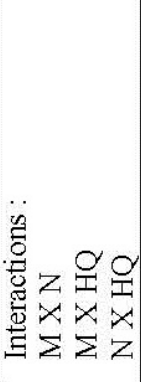 \\
\hline
\end{tabular}

Egypt. J. Agron. 34, No. 1 (2012) 
RESPONSE OF TWO MAIZE HYBRIDS ...

\begin{tabular}{|c|c|c|c|c|c|}
\hline 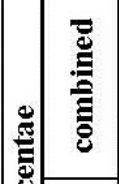 & 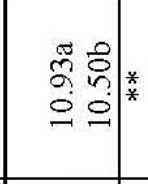 & $\begin{array}{lll}\text { Nิ } & 0 \\
& 0 \\
0 & 0 & 0 \\
- & 0\end{array}$ & 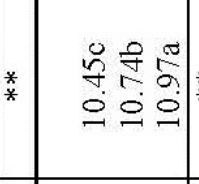 & & 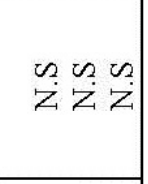 \\
\hline 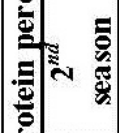 & 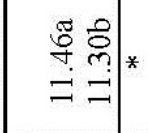 & 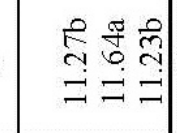 & 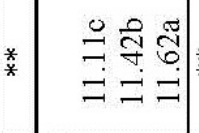 & & 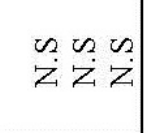 \\
\hline$=\frac{5}{2}$ & 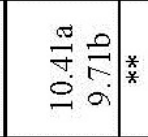 & $\begin{array}{lll}8 & 0 \\
8 & 8 & 0 \\
\circ & 0 & 0\end{array}$ & 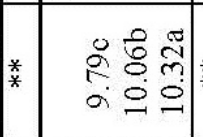 & $\div$ & $\begin{array}{lll}* & 02 \\
* & z & z \\
& z\end{array}$ \\
\hline 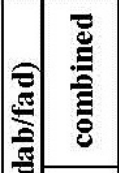 & 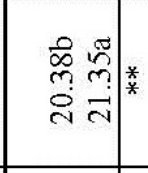 & 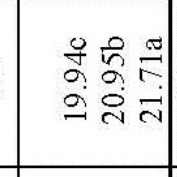 & 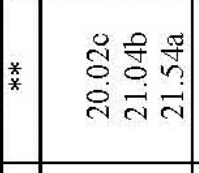 & & $\begin{array}{l}\mathscr{O}_{2}^{2}{ }^{*} \\
Z_{Z}{ }^{2}\end{array}$ \\
\hline 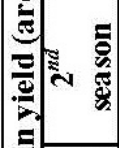 & 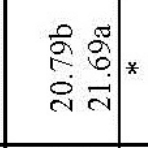 & 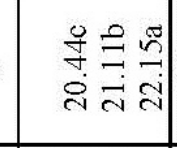 & 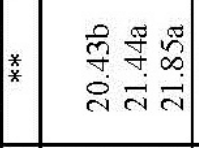 & & 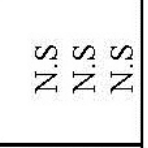 \\
\hline 証 & 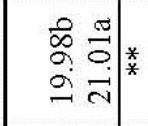 & 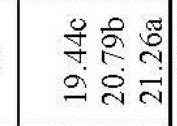 & 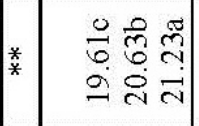 & & $\begin{array}{l}C_{2}^{2} * \\
Z Z^{*} *\end{array}$ \\
\hline 绨 & 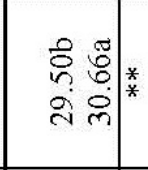 & 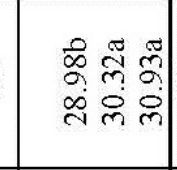 & 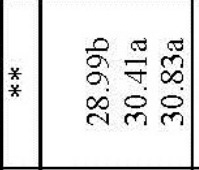 & & 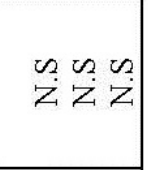 \\
\hline तै & 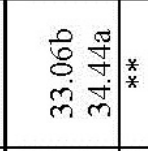 & 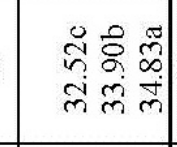 & 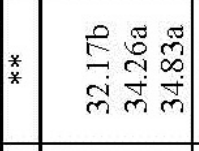 & $\begin{array}{l}\infty \\
z\end{array}$ & 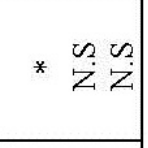 \\
\hline 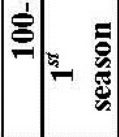 & 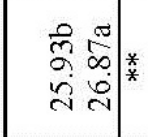 & 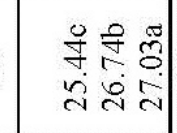 & 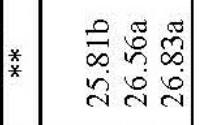 & & 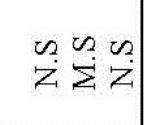 \\
\hline 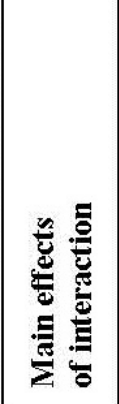 & 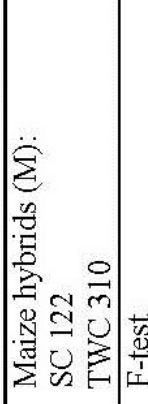 & 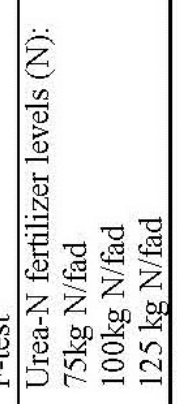 & 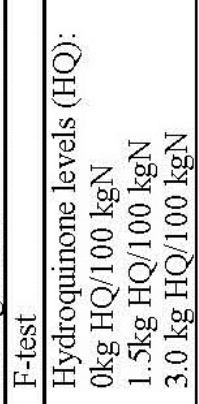 & & 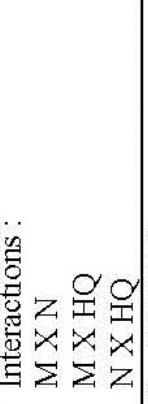 \\
\hline
\end{tabular}

Egypt. J. Agron. 34, No. 1 (2012) 


\begin{tabular}{|c|c|c|c|c|c|}
\hline 馬 & 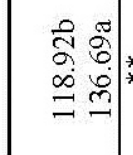 & 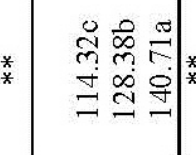 & 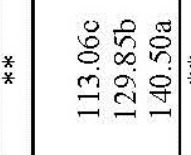 & & 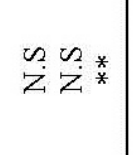 \\
\hline 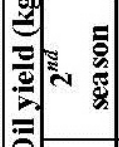 & 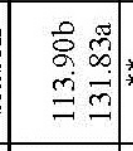 & 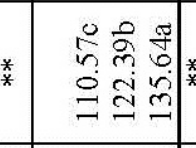 & 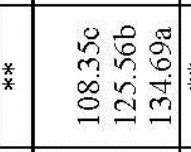 & & 兄* \\
\hline 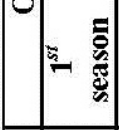 & 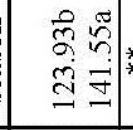 & 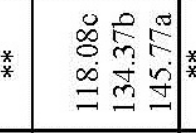 & 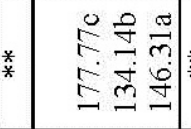 & & $\begin{array}{l}\sim_{2}^{2} * * \\
\dot{z} z^{*} *\end{array}$ \\
\hline 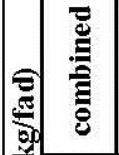 & 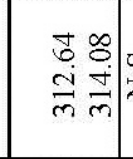 & 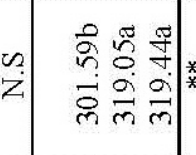 & 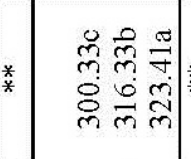 & & 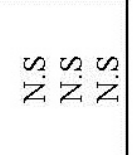 \\
\hline 政 & 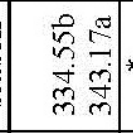 & 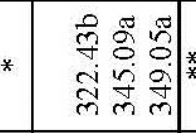 & 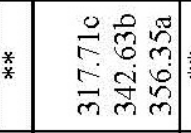 & & 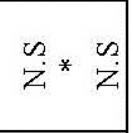 \\
\hline 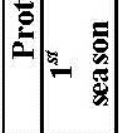 & 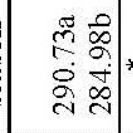 & 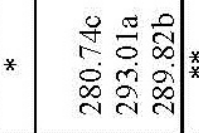 & 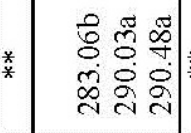 & & 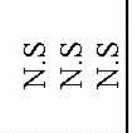 \\
\hline 焉 & 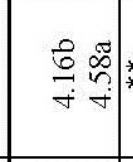 & 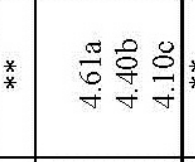 & 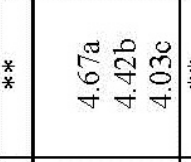 & $*$ & 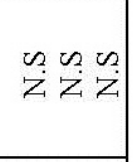 \\
\hline 咅: & 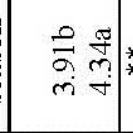 & 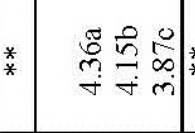 & 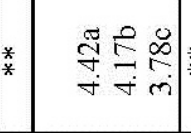 & & 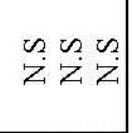 \\
\hline 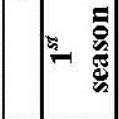 & 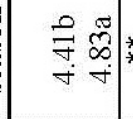 & 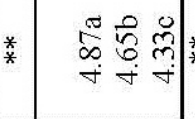 & 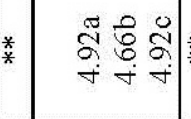 & & $\underset{z}{2} \sum_{i}^{*}$ \\
\hline 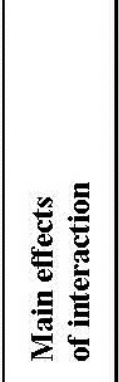 & 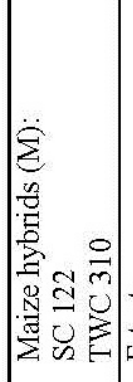 & 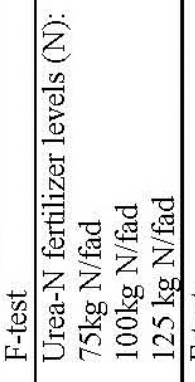 & 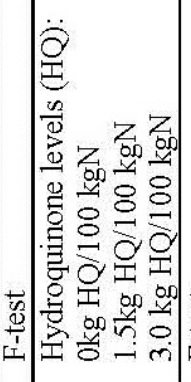 & 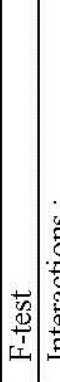 & 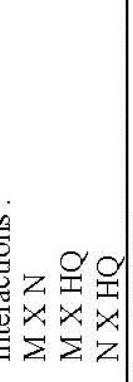 \\
\hline
\end{tabular}

Egypt. J. Agron. 34, No. 1 (2012) 
Urease inhibitor $(H Q)$ effects

The amendment of urea fertilizer with HQ as a urease inhibitor caused significant increases in yield and all yield attributes characters of maize, except oil percentage and this was more pronounced in the combined analysis of the two growing seasons. Ear length, number of ears/plant, number of rows/ear, number of grains/row, number of grains /ear, protein percentage, grain, protein and oil yields/fad were continuously and significantly increased by treating urea with HQ urease inhibitor up to $3.0 \mathrm{~kg} / \mathrm{fad}$, whereas both plant height and 100 -grain weight were significantly responded to $1.5 \mathrm{~kg} \mathrm{HQ} / \mathrm{fad}$.

Compared to check plots (without HQ application), each increment of HQ urease inhibitor resulted in a mean increase of 0.760 ardab, $11.540 \mathrm{~kg}$ and $13.720 \mathrm{~kg}$ for grain, protein and oil yields/fad, respectively. The beneficial effects of treating urea with HQ urease inhibitor may be attributed to reducing urea-N losses and hence increasing fertilizer use efficiency as well as the important role of HQ to retard urea hydrolysis and reduced gaseous and leaching losses and thereby maintained an optimal level of available $\mathrm{N}$ in the soil for a long period (Khamis et al., 2005). Shlegel et al. (1986) reported that urease inhibitors increased grain yield of maize when added to urea that was surface-applied. Similar results were reported by Zhang et al. (1997) and Hernan et al. (1999 and 2001).

\section{Interaction effects}

Data in Table 5 revealed that TWC 310 always gave the higher number of grains/ ear compared to SC 122 and this was true under the three levels of HQ urease inhibitor. The number of grains/ ear was responded to HQ levels up to $1.5 \mathrm{~kg} / \mathrm{fad}$ and up to $3.0 \mathrm{~kg} / \mathrm{fad}$ regarding to SC 122 and TWC 310, respectively. Again, the higher increase due to HQ increment was obtained from TWC 310 where each increment of HQ produced an increase of 25.662 grains/ear compared to SC 122 which gave an increase in grains number / ear of 17.294 due to each HQ increment.

TABLE 5. The interaction effect between maize hybrids and HQ levels on number of grains/ear (combined).

\begin{tabular}{|l|c|c|c|c|}
\hline \multirow{2}{*}{ Maize hybrids } & \multicolumn{3}{|c|}{ HQ levels (kg/100kg N) } & \multirow{2}{*}{$\begin{array}{c}\text { Response } \\
\text { rate }\end{array}$} \\
\cline { 2 - 4 } & $\mathbf{0}$ & $\mathbf{1 . 5}$ & $\mathbf{3 . 0}$ & \\
\hline \multirow{3}{*}{ SC 122} & $\mathrm{~B}$ & $\mathrm{~A}$ & A & \\
TWC 310 & $476.828 \mathrm{~b}$ & $497.794 \mathrm{~b}$ & $\begin{array}{c}511.417 \mathrm{~b} \\
\mathrm{~A}\end{array}$ & 17.294 \\
& $526.444 \mathrm{a}$ & $555.878 \mathrm{a}$ & $577.767 \mathrm{a}$ & 25.662 \\
\hline
\end{tabular}

The interaction effect between urea-N and HQ levels on grain and oil yields/ fad was significant as shown in Table 6. At the three studied levels of nitrogen, grain yield was responded to HQ urease inhibitor up to $1.5 \mathrm{~kg} / \mathrm{fad}$. When urea untreated with HQ, grain yield / fad significantly increased up to $125 \mathrm{~kg} \mathrm{~N} / \mathrm{fad}$, 
but when applying $\mathrm{HQ}$ at the rate of 1.5 or $3.0 \mathrm{~kg} / \mathrm{fad}$, the grain yield / fad responded to $100 \mathrm{~kg} \mathrm{~N} / \mathrm{fad}$.

TABLE 6. The interaction effect between $\mathbf{N}$ fertilizer and HQ levels on grain and oil yields / fad of maize (combined).

\begin{tabular}{|c|c|c|c|c|}
\hline \multirow{3}{*}{$\begin{array}{l}\text { N-fertilizer levels } \\
\text { (kg N/fad) }\end{array}$} & \multicolumn{3}{|c|}{ HQ levels $(\mathrm{kg} / 100 \mathrm{~kg} \mathrm{~N})$} & \multirow{3}{*}{$\begin{array}{l}\text { Response } \\
\text { rate }\end{array}$} \\
\hline & $\mathbf{0}$ & 1.5 & 3.0 & \\
\hline & \multicolumn{3}{|c|}{ Grain yield (ardab/fad) } & \\
\hline 75 & $\begin{array}{c}\mathrm{B} \\
19.221 \mathrm{c} \\
\mathrm{B}\end{array}$ & $\begin{array}{c}A \\
20.091 b \\
A\end{array}$ & $\begin{array}{c}\mathrm{A} \\
20.508 \mathrm{c} \\
\mathrm{A}\end{array}$ & 0.644 \\
\hline 100 & $\begin{array}{c}20.062 b \\
B\end{array}$ & $\begin{array}{c}21.156 a \\
\mathrm{AB}\end{array}$ & $\begin{array}{c}21.637 \mathrm{a} \\
\mathrm{A}\end{array}$ & 0.788 \\
\hline 125 & $20.780 \mathrm{a}$ & $21.858 \mathrm{a}$ & $22.476 \mathrm{a}$ & \multirow[t]{2}{*}{0.848} \\
\hline Response rate & 0.780 & 0.884 & 0.984 & \\
\hline \multicolumn{5}{|c|}{ Oil yield $(\mathrm{kg} / \mathrm{fad})$} \\
\hline 75 & $\begin{array}{c}\mathrm{C} \\
103.599 \mathrm{~b} \\
\mathrm{C}\end{array}$ & $\begin{array}{c}\mathrm{B} \\
115.726 \mathrm{c} \\
\mathrm{B}\end{array}$ & $\begin{array}{c}\mathrm{A} \\
123.647 \mathrm{c} \\
\mathrm{A}\end{array}$ & \multirow{4}{*}{$\begin{array}{c}10.024 \\
9.684 \\
21.462\end{array}$} \\
\hline 100 & $118.879 \mathrm{a}$ & $\begin{array}{c}128.022 b \\
B\end{array}$ & $\underset{\mathrm{A}}{138.247 \mathrm{~b}}$ & \\
\hline 125 & $116.694 \mathrm{a}$ & $145.804 \mathrm{a}$ & $159.617 \mathrm{a}$ & \\
\hline Response rate & 6.548 & 15.039 & 17.985 & \\
\hline
\end{tabular}

Without HQ application, urea-N applied at $100 \mathrm{~kg} \mathrm{~N} / \mathrm{fad}$ produced the highest oil yield/ fad, while when urea fertilizer treated with 1.5 or $3.0 \mathrm{~kg} \mathrm{HQ} / \mathrm{fad}$, the oil yield/ fad was increased up to $125 \mathrm{~kg} \mathrm{~N} / \mathrm{fad}$. At the three levels of urea-N, oil yield/fad was remarkably increased with increasing HQ urease inhibitor level up to $3.0 \mathrm{~kg} / \mathrm{fad}$ but with different magnitudes. The highest increase due to HQ increment was obtained from $125 \mathrm{~kg} \mathrm{~N} /$ fad where each increment of HQ produced an increase of $21.462 \mathrm{~kg}$ of oil yield. Only about 10.024 and $9.684 \mathrm{~kg}$ of oil yield was produced from adding 75 and $100 \mathrm{~kg} \mathrm{~N} /$ fad, respectively.

\section{Yield analysis}

Correlation study

Table 7 clearing simple correlation coefficients between maize grain yield/fad and other studied characters (pooled data of both seasons). Positive and significant correlation coefficients were found between grain yield/ fad and each of plant height, ear length , number of ears/ plant, number of rows/ear, number of grains/ear, 100-grain weight and protein and oil yields/fad, while grain yield/ fad was negatively and significantly correlated with oil percentage. However, grain yield was positively and insignificantly correlated with protein percentage. Data clearly indicate that the correlation coefficients between any pair of studied characters were positive and significant, except the correlation between protein percentage and most of other studied characters which was positive and insignificant as well as between protein yield/fad and each of ear length, number of rows/ ear, number of grains/ row and number of grains/ ear which was positive and insignificant. Oil percentage was negatively and significantly correlated with most of studied traits.

Egypt. J. Agron. 34, No. 1 (2012) 


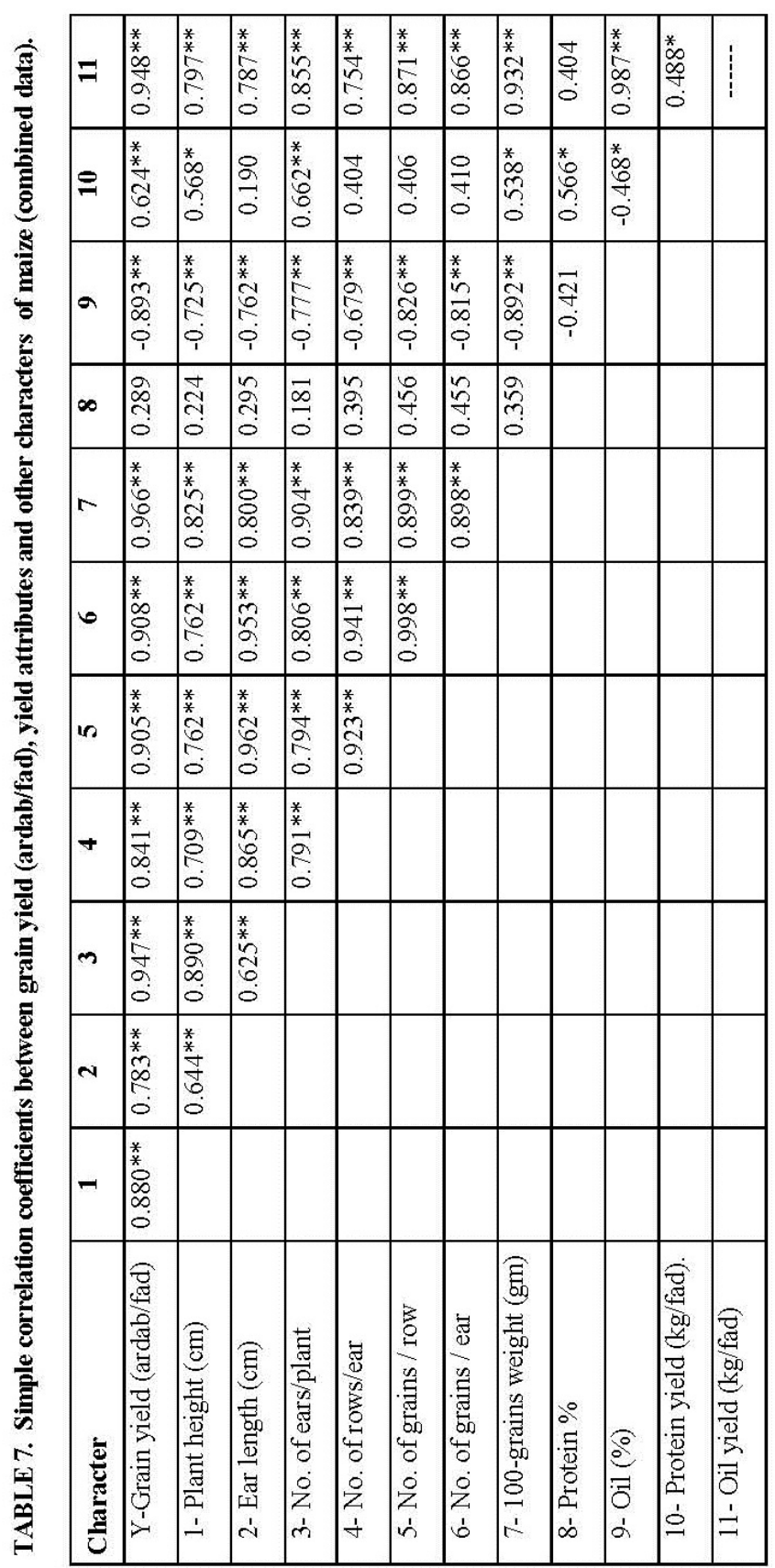

Egypt. J. Agron. 34, No. 1 (2012) 
Path analysis

The partitioning of simple correlation coefficient between maize grain yield and its components mentioned herein is presented in Table 8.

TABLE 8. Partioning of simple correlation coefficients between maize grain yield (ardab/fad) and its attributes.

\begin{tabular}{|l|c|}
\hline & \\
Sources & Values \\
& \\
\hline Number of ears/plant: & \\
Direct effect & 0.4130 \\
Indirect effect via number of grains /ear & 0.1817 \\
Indirect effect via 100-grain weight & 0.3523 \\
Total (ry $)$ & 0.9470 \\
\hline Number of grains / ear: & \\
Direct effect & 0.2260 \\
Indirect effect via number of ears / plant & 0.3321 \\
Indirect effect via 100-grain weight & 0.3499 \\
Total (ry ) $^{\text {) }}$ & 0.9080 \\
\hline 100-grain weight: & \\
Direct effect & 0.3897 \\
Indirect effect via number of ears / plant & 0.3734 \\
Indirect effect via number of grains / ear & 0.2029 \\
Total (ry ${ }_{3}$ ) & 0.9660 \\
\hline
\end{tabular}

Data clearly show that the number of ears / plant had the highest direct effect on grain yield (0.4130) followed by 100 -grain weight $(0.3897)$ and number of grains / ear (0.2260) in a descending order. Also, the indirect interaction effects between each pair of mentioned components on grain yield gave a considerable values in this respect.The relative importance in contributing grain yield / fad of maize as a percentage of the variation of number of ears / plant, number of grains/ ear and 100-grain weight with their interactions is listed in Table 9 . The results indicate that the number of ears/plant gave a relative contribution of $17.06 \%$ of the grain yield variation and being higher than that of 100-grain weight and number of grains /ear which was 15.18 and $5.11 \%$ of the grain yield variation, respectively. It is worthy to observe that the joint effect of number of ears/plant with number of grains/ear and with 100-grain weight; number of grains/ear with 100-grain weight as $15.01,29.10$ and $15.82 \%$ of the variation, respectively. Here, it is worthy to note that those mentioned characters as well as their interactions could contributed much in maize grain yield since $\mathrm{R}^{2}$ was $97.28 \%$ of the total variation in yield. Also, it is interesting to observe that the residual effects contributing to grain yield in this study was low in magnitude being $2.72 \%$. 
TABLE 9. Direct and joint effects of grain yield attributes presented as a percentage of grain yield variation of maize.

\begin{tabular}{|l|c|c|}
\hline Sources & C.D. & \% \\
\hline Number of ears/plant & 0.1706 & 17.06 \\
Number of grains /ear & 0.0511 & 5.11 \\
100-grain weight & 0.1518 & 15.18 \\
Number of ears/plant X Number of grains/ear & 0.1501 & 15.01 \\
Number of ears/plant X 100-grain weight & 0.2910 & 29.10 \\
Number of grains/ears X 100-grain weight & 0.1582 & 15.82 \\
$\mathrm{R}^{2}$ & 0.9728 & 97.28 \\
Residual & 0.0272 & 2.72 \\
Total & $\mathbf{1 . 0 0 0 0}$ & $\mathbf{1 0 0 . 0 0}$ \\
\hline
\end{tabular}

C.D. = Coefficient of determination

$\%=$ Percentage contributed

Regression study

Parameters of regression analysis between urea- $\mathrm{N}$ rates and maize grain yield as well as yield components are presented in Table 10.

Data clearly indicate that the response of number of ears/ plant and number of grains / ear are similar to that of grain yield /fad and each is of quadratic relationship with urea-N application rates. While, the response of 100grain weight had a linear relationship with urea-N application rates. Here, it is worthy to note that maize grain yield could be increased by means of increasing 100-grain weight if urea- $\mathrm{N}$ is applied at suitable dose and at the proper age of plant.

TABLE 10. Parameters of regression analysis between urea-N fertilization and grain yield as well as yield attributes of maize.

\begin{tabular}{|l|c|c|c|c|}
\hline Parameters & $\begin{array}{c}\text { Number of } \\
\text { ears/plant }\end{array}$ & $\begin{array}{c}\text { Number of } \\
\text { grains/ear }\end{array}$ & $\begin{array}{c}\text { 100-grain weight } \\
\text { (gm) }\end{array}$ & $\begin{array}{c}\text { Grain yield } \\
\text { (ardab/fad) }\end{array}$ \\
\hline a & 1.13 & 453.40 & 27.34 & 18.95 \\
b & 8.515 & 1.610 & 4.169 & 1.083 \\
c & -1.034 & -0.535 & 2.500 & -0.413 \\
$\mathrm{R}^{2}$ & 0.9471 & 0.9041 & 0.9080 & 0.9483 \\
Max. X & 3.61 & 3.25 & - & 3.45 \\
Max. Y & 1.41 & 604.50 & - & 22.81 \\
\hline
\end{tabular}

\section{Conclusion}

It could be concluded that the highest grain yield/fad of TWC 310 maize hybrid could be attained by adding $100 \mathrm{~kg} \mathrm{~N} /$ fad with 1.5 or $3.0 \mathrm{~kg}$ HQ urease inhibitor / $100 \mathrm{~kg} \mathrm{~N}$ to decrease about $25 \mathrm{kgN}$ of applied nitrogen amount under loamy sand soil conditions at Sharkia Governorate. 


\section{References}

A.O.A.C (1988) "Official Methods of Analysis". Association of Official Analytical Chemists, $21^{\text {st }}$ ed., Washington, D.C., USA.

Abd El-Maksoud, M.F and Sarhan, A.A. (2008) Response of some maize hybrids to bio and chemical nitrogen fertilization. Zagazig J. Agric. Res. 35 (3) , 497 - 515.

Ahmed, M.A. and El-Sheikh, M.H. (2002) Response of maize cultivars to different management regimes. J. Agric. Sci. Mansoura Univ. 29 (8), 4821 - 4833.

Aly, A.M., Badr, S.K. and Greish, M.H.M. (1996) Effect of variety, plant population and nitrogen application on grain yield of two maize varieties. Proc. $7^{\text {th }}$. Conf. Agronomy, 9-10 Sept., pp.71-80.

Comstock, V.E. and Culberston, J.O. (1958) A rapid method of determining the oil content and iodine values. Agron. J. 50 (1), 113 - 114.

Duncan, D.B. (1955) Multiple range and multiple F-test. Biometerices, 11, 1 - 42.

Hans, B. (2006) Nitrogen fertilization, yield and protein quality of a normal and a high lysine maize variety. J. Sci. Food Agric. 27 (10), 978 - 982

Hernan, R., Hernan, E., Studdert, G.H. and Andrade, F.A. (1999) No-till maize nitrogen uptake and yield: Effect of urease inhibitor and application time. Agron. J. 91, 950 - 955 .

Hernan, R., Hernan, E. and Picone, L. (2001) Denitrification in maize under no-tillage: Effect of nitrogen rate and application time. Soil Sci. Soc. AM. J. 65, 1314 - 1323.

Khamis, M.A., Metwally, Sh. M. and Gaballah, A.B. (2005) A new urease inhibitor (1, 4 - phenylendiamine): 2 - Effect on maize production and fertilizer use efficiency. J. Product. \& Dev. 19 (2), 371 - 384.

Mohamed, N.A. (2004) Principal component and response curve analysis of some maize hybrids to different fertilization levels and plant density. Bull. Fac. Agric., Cairo Univ. 55, 531- 556 .

Oraby, F.T., Abd El-Maksoud, M.F. and Sarhan, A.A. (2005) Proper agronomic practices required to maximize productivity of some maize varieties in old and reclaimed soils. V-Response of ten maize hybrids to $\mathrm{N}$ fertilization under two locatinos. J. Product. \& Dev. 10 (1), 55 - 73.

Svab, J. (1973) Biometrial modszerek a kutatas ban-Mezo-gazassagi. Kiado, Budapest (C.F. Sunflower Conf., July, Novi Sad, Vol. 1: pp.423 - 428).

Shlegel, A.J., Nelson, D.W. and Sommers, L.E. (1986) Field evaluation of urease inhibitors for corn production. Agron. J. 78, 1007- 1012.

Thiraporn, R., Geisler, G. and Stamp, P. (2008) Effect of nitrogen fertilization on yield and yield components of tropical maize cultivars. J. Agron . Crop Sci. 159 (1), 9 - 14.

Egypt. J. Agron. 34, No. 1 (2012) 
Vetsch, J.A. and Randall, G.W. (2000) Enhancing no - tillage systems for corn with starter fertilizers, row cleaner and nitrogen placement methods. Agron. J. 92, 309 - 312.

Zhang, Z., Li, J., Feng, Y., Shuchun, B. and Weimin, Wu. (1997) Physical and chemical properties of a durably efficacious ammonium bicarbonate as a fertilizer and its yield - increasing mechanism. Chinese Acad. Sci. 40 (1), 105 - 112.

(Received 28/3/2012

$$
\begin{aligned}
& \text { إستجابة صنفين من الذرة الشامية للتسميد باليوريا تحت تأثير } \\
& \text { إضافة مثبط إنزيم اليوريز الهنيد الئروكينون الثيامين }
\end{aligned}
$$

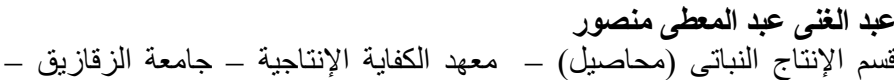

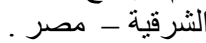

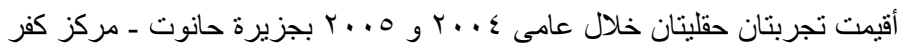

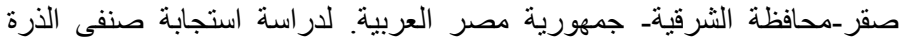

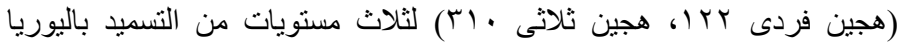

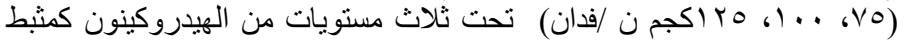

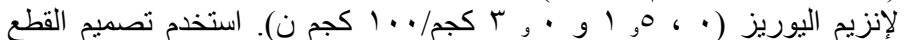

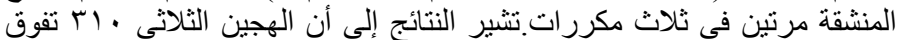

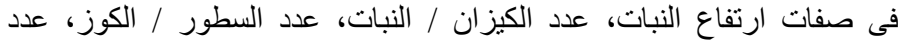

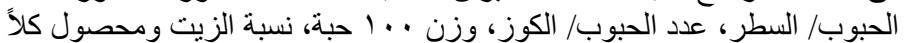

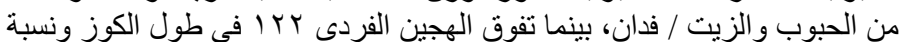

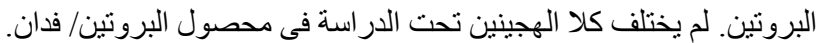

أدت زيادة مستوى التسميد باليوريا من Vo إلى . . 1 كجم ن / فدان إلى زيادة

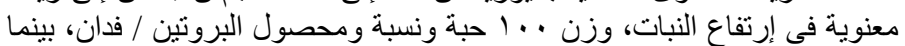

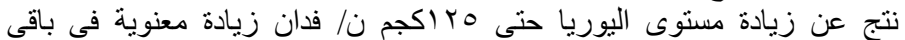
الصفات المدروسة ما عدا نسبة الزيت التى انخفضت مع زيادة مستوى اليوريا.

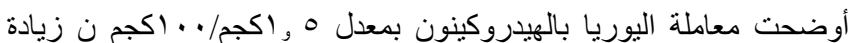

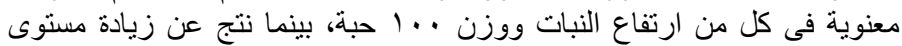

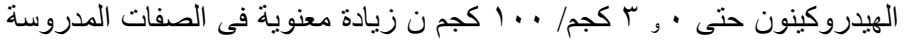

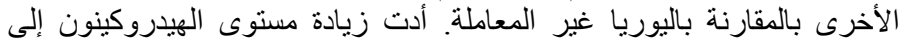
نقص معنوى فى نسبة الزيت.

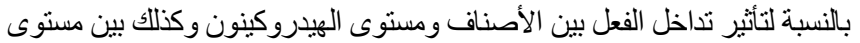

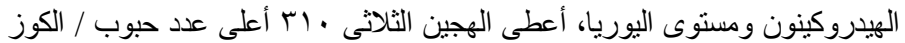

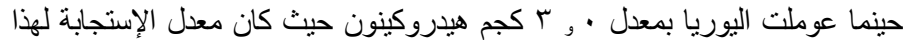

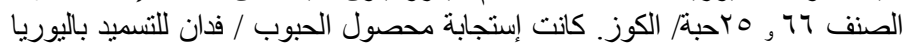

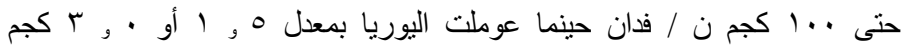




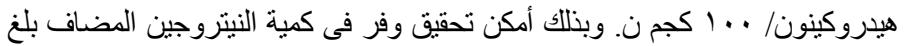

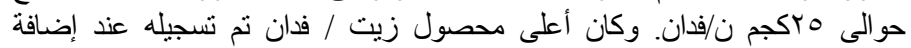

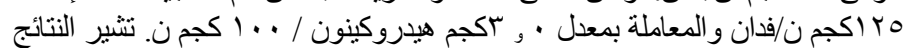

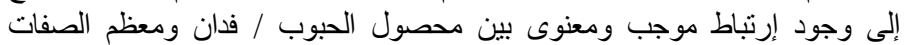

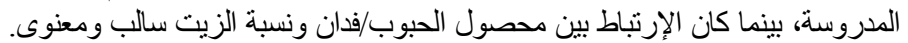

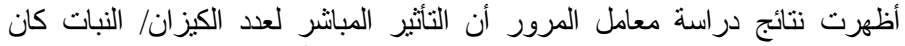

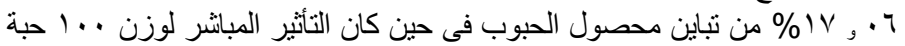

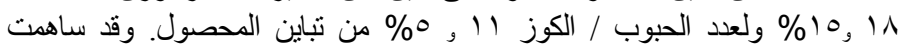

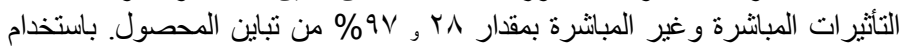

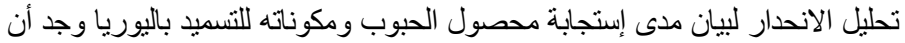

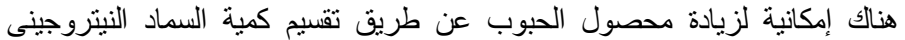
المناسبة لكى تكون أكثر فعالية فى زيادة وزن ل . ل.

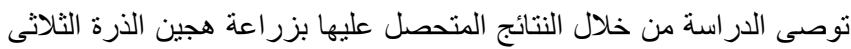

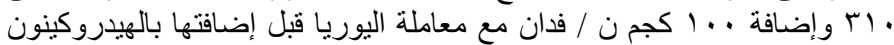

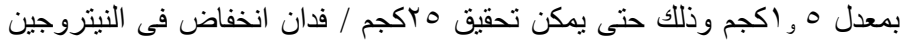

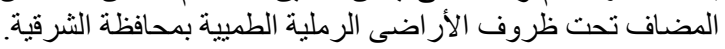

